\title{
Perfil das perdas dentárias em adultos segundo o capital social, características demográficas e socioeconômicas
}

Adult tooth loss profile in accordance with social capital and demographic and socioeconomic characteristics

Departamento de Odontologia Social e Preventiva, Faculdade de Odontologia, Universidade Federal de Minas Gerais. Av. Presidente Antônio Carlos 6627, Pampulha.

31.270-010 Belo Horizonte MG Brasil.

carolmborges@yahoo.com.br
Abstract Demographic and socioeconomic conditions play an important role in tooth loss in the population, however, there is little scientific evidence regarding the influence of social capital on this outcome. The scope of this study was to describe the tooth loss profile of adults aged 35-44, who are residents of the surrounding area of the city of Belo Horizonte in the state of Minas Gerais. This cross-sectional exploratory study comprised a sample of 1,013 adults. The dependent variable was tooth loss. Exploratory variables were social capital, demographic and socioeconomic characteristics. The Chi-squared Automatic Interaction Detector (CHAID) was used to map the adult tooth loss profile which was explained by low levels of social capital (47.0\%; $p<0.001)$, more elderly adults (60.2\%; $p<0.001)$, and low education levels $(66.3 \% ; p=0.022)$. In the high social capital group, age was the determinant factor of tooth loss, irrespective of educational level or per capita income. The conclusion, at the individual level, is that social capital as well as demographic and socioeconomic characteristics explained the adult tooth loss profile. Individual social capital may have attenuated the negative influence of socioeconomic factors in the population under scrutiny.

Key words Tooth loss, Oral health, Epidemiology, Social determinants, Decision tree
Resumo Condições socioeconômicas e demográficas exercem um importante papel nas perdas dentárias da população, no entanto existem poucas evidências científicas sobre a influência do capital social nesse agravo. O objetivo deste estudo foi descrever o perfil das perdas dentárias em adultos de 35-44 anos de idade, residentes em municípios do entorno de Belo Horizonte, Minas Gerais. Este estudo transversal exploratório analisou dados referentes a 1.013 indivíduos. A variável dependente foi a perda dentária. As variáveis exploratórias foram o capital social e as características demográficas e socioeconômicas. A análise de árvore de decisão por meio do Algoritmo Chi-squared Automatic Interaction Detector (CHAID) foi utilizada para mapear o perfil das perdas dentárias, a qual foi explicada pelo baixo capital social (47,0\%; $p<0,001)$, adultos mais velhos $(60,2 \% ; p<0,001)$ e baixa escolaridade $(66,3 \% ; p=0,022)$. No grupo com alto capital social, a idade foi o fator determinante das perdas dentárias, independente da escolaridade ou renda per capita. Conclui-se que, no nível individual, o capital social, a idade e a renda explicaram o perfil das perdas dentárias. $O$ capital social individual pode ter atenuado o efeito negativo dos fatores socioeconômicos na população estudada.

Palavras-chave Perda dentária, Saúde bucal, Epidemiologia, Determinantes sociais, Árvore de decisão 


\section{Introdução}

A condição de saúde bucal dos adultos brasileiros melhorou, de maneira geral, nos últimos vinte e cinco anos. Esse fato pode ser constatado pela comparação dos resultados dos inquéritos epidemiológicos nacionais para avaliar a saúde bucal da população realizados no Brasil. Em 1986, o índice CPOD (composto pela soma de dentes cariados, extraídos e restaurados) era de 22,5 na população adulta com idade entre 35 e 44 anos tendo o componente $\mathrm{P}$ (número de dentes extraídos) contribuído com $66 \%$ deste total ${ }^{1}$. Após duas décadas, o perfil de morbidade se manteve praticamente inalterado; a média do CPOD em adultos foi de 20,1 com contribuição de $65,7 \%$ do componente perdido ${ }^{2}$. Em nota técnica divulgada na imprensa, o Ministério da Saúde apresentou os principais resultados do último levantamento nacional de saúde bucal da população brasileira, o SB-Brasil 2010. O ataque de cárie à população adulta de 35-44 anos reduziu nestes últimos sete anos comparado aos resultados do inquérito anterior. O número médio do $\mathrm{CPOD}$ declinou de 20,1 para 16,3 - redução do componente perdido de $65 \%$ para $43,75 \%{ }^{3}$.

A principal causa das perdas dentárias em adultos é a doença cárie não tratada ${ }^{4,5}$. A doença periodontal também se associa às perdas dentárias; estudos apontam que o uso do tabaco é o maior fator de risco para a doença periodontal nos adultos. A doença periodontal quando não tratada pode contribuir com o aumento do número de perdas dentárias ${ }^{6,7}$. O traumatismo dentário $^{8} \mathrm{e}$ a atitude profissional não conservadora ${ }^{9}$ também podem levar às perdas dentárias, porém em menor número quando comparadas às demais causas.

No que tange à determinação social das perdas dentárias, as características demográficas e socioeconômicas desempenham um papel importante na distribuição desse agravo na população. O número de dentes perdidos em adultos brasileiros é maior entre os mais velhos, do gênero feminino, residentes nas zonas rurais, mais pobres e com menor escolaridade ${ }^{10}$. Resultado de estudo semelhante com dados nacionais reforçou a importância do contexto social como fator fortemente associado às perdas dentárias nessa faixa-etária, enfatizando a alta escolaridade como proteção contra o agravo ${ }^{11}$. Estudo de base populacional sobre o número de dentes remanescentes em adultos de 35-44 anos residentes no estado de São Paulo corrobora com os achados referentes aos dados nacionais ${ }^{12}$.
As perdas dentárias podem causar impacto negativo tanto na qualidade de vida das pessoas quanto na própria sociedade onde estão inseridas. No que tange à primeira questão, a falta dos dentes pode comprometer a vida diária dos indivíduos devido à dificuldade na fala, limitação da mastigação, ao constrangimento, à timidez, à diminuição da autoestima e à exclusão social ${ }^{13,14}$. Quanto ao impacto na sociedade, os serviços de saúde ainda não conseguem atender a demanda acumulada de tratamento odontológico reabilitador $^{15}$. O aumento da necessidade de próteses dentárias implica aumento no custo do tratamento. Em 2010, cerca de 69\% dos adultos brasileiros necessitavam de algum tipo de prótese dentária ${ }^{3}$.

Ainda que incipientes, os estudos do capital social - que se refere às características da estrutura social como normas e valores que podem beneficiar indivíduos e facilitar ações coletivas ${ }^{16,17}$ - e as perdas dentárias apontam o baixo capital social como fator de risco para o número de dentes perdidos em idosos ${ }^{18}$. Até a presente data, não há evidências conclusivas sobre a associação entre o capital social e a saúde bucal devido à inexistência de estudos longitudinais.

Apesar de a perda dentária ser considerada uma medida de baixos níveis de saúde bucal, há poucas publicações científicas brasileiras sobre esse agravo e possíveis fatores associados em adultos $^{10}$. O objetivo deste estudo foi traçar um perfil das perdas dentárias segundo o capital social, características demográficas e socioeconômicas em adultos de 35-44 anos de idade, residentes nos municípios do entorno de Belo Horizonte, Minas Gerais, Brasil.

\section{Métodos}

O presente estudo transversal exploratório foi realizado entre os meses de Maio e Dezembro de 2010. A população de referência foi composta por 88.958 adultos de 35-44 anos de idade, residentes na zona urbana do entorno de Belo Horizonte, Minas Gerais, composto por 33 municípios. O método utilizado para o cálculo amostral foi o de comparação de duas proporções. A perda dentária foi dicotomizada em $\leq 12 \mathrm{e}>12$ dentes per$\operatorname{didos}^{10,19}$ e o indicador de capital social medido como a participação em atividades comunitárias ( $\geq 01$ vez; nenhuma vez) nos últimos 12 meses anteriores à entrevista. A prevalência de $>12$ perdidos foi de $18,2 \%$ entre aqueles que não participaram de nenhuma atividade comunitária e $10,0 \%$ entre os que afirmaram ter participado 
pelo menos uma vez (diferença de 8,2\%). A amostra mínima requerida foi de 1.116 adultos (assumindo-se poder $=90 \%$, nível de significância de $5 \%$, acréscimo de $20 \%$ para compensar possíveis perdas e deff igual a 2.0 para corrigir a perda da variabilidade da amostra).

Para a seleção da amostra, verificou-se a distribuição do número de habitantes de cada um dos 33 municípios, sendo, agrupados segundo quartis de porte populacional. Dois municípios de cada estrato populacional foram sorteados aleatoriamente. A amostragem por cluster foi utilizada para selecionar quadras em municípios com até 50 mil habitantes (Cluster I) e setores censitários e quadras em municípios com mais de 50 mil habitantes (Cluster II).

O número de adultos examinados foi distribuído proporcionalmente ao número total de adultos residentes em cada um dos municípios sorteados. Todas as residências localizadas nas quadras sorteadas foram visitadas e todos os adultos pertencentes ao grupo etário investigado foram examinados.

O critério de inclusão foi ter nascido entre 1966 e 1975 na data do exame/entrevistas. Os exames clínicos e as entrevistas foram realizados em domicílio. As entrevistas foram conduzidas com auxílio de formulários estruturados. Todos os procedimentos relacionados aos exames clínicos seguiram as recomendações da Organização Mundial da Saúde para levantamentos epidemiológicos ${ }^{20}$. A calibração dos cinco examinadores foi satisfatória. A média do Teste Kappa interexaminadores para o Índice CPOD foi de 0,853 [0,81$0,92]$ e 0,884 [0,85-1,00] intraexaminadores.

\section{Variáveis investigadas}

A variável dependente foi a perda dentária medida pelo componente "perdido" do Índice CPOD, dicotomizada pela mediana $(\leq 4 \mathrm{e} \geq 5$ dentes perdidos). Apesar de o estudo piloto ter considerado um recorte dessa variável igual $\leq 12$ e $>12^{10}$, não se mostrou apropriado para a análise do estudo principal uma vez que a média do número de dentes perdidos foi de 5,3, justificando a escolha pela mediana.

Os indicadores utilizados como proxy de capital social foram os que seguem: 1) participação em grupos formais ou informais ( $\geq 01$; nenhum), 2) participação em algum projeto comunitário nos últimos doze meses anteriores à entrevista (sim; não), 3) sentimento de segurança quando está em casa sozinho (sim - muito seguro/ seguro; talvez; não - muito inseguro/ inseguro) e 4) intenção de voluntariar (sim; não) - doação de tempo em projetos comunitários ${ }^{21}$.

Para criar a variável binária capital social, foi utilizada a análise de segmentação (cluster) com validação da divisão dos grupos por meio de análise discriminante dos quatro proxies de capital social (participação grupos $\mathrm{p}=0,046 /$ participação projeto comunitário $\mathrm{p}=0,131 /$ sentimento segurança $\mathrm{p}<0,001 /$ intenção voluntariar $\mathrm{p}=$ 0,056), resultando em dois grupos (alto e baixo capital social) - dados não apresentados. A análise de segmentação é uma ferramenta estatística utilizada para agrupar dados, alocando os casos mais similares no mesmo grupo com base nas características medidas entre os participantes do estudo $^{22}$. Empregou-se o método K-Mean Cluster a fim de medir a contribuição de cada variável na formação dos clusters por meio do teste de ANOVA.

As características demográficas e socioeconômicas investigadas foram a idade dicotomizada pela mediana ( $\leq 40 ;>40$ anos), o gênero (masculino; feminino), a cor da pele atuorrelatada (branca, parda, amarela, preta, indígena), o estado civil (casado, divorciado/separado, viúvo, solteiro), a renda familiar per capita obtida por meio da razão entre o número total de moradores da residência e a renda familiar mensal e, então, dicotomizada pela mediana ( $>\mathrm{R} \$ 300,00$; $\leq$ $\mathrm{R} \$ 300,00)$ e escolaridade categorizada (graduação - pós-graduação/graduação completa/graduação incompleta; ensino médio - completo/ incompleto; ensino fundamental - completo/incompleto; analfabeto).

Este estudo foi aprovado pelo Comitê de Ética em Pesquisa da Universidade Federal de Minas Gerais.

\section{Análise dos dados}

Para traçar o perfil das perdas dentárias na população estudada, optou-se pela técnica da árvore de decisão que apresenta os fatores associados ao problema investigado apresentando uma ordem de priorização. Esse método consiste em regras de decisão que realizam sucessivas divisões no conjunto de dados de forma a tornálo cada vez mais homogêneo em relação à variável desfecho. A árvore de decisão se apresenta sob a forma de um gráfico que começa com um nó raiz, onde todas as observações da amostra são apresentadas. Os nós produzidos em sequência representam subdivisões dos dados em grupos cada vez mais homogêneos ${ }^{22}$. O modelo foi ajustado mediante sucessivas divisões binárias 
(nós) nos conjuntos de dados. O critério de parada foi o valor $\mathrm{p} \leq 0,05$ da estatística qui-quadrado usando a correção de Bonferroni. $\mathrm{O}$ ajuste do modelo final foi avaliado pela estimativa de risco geral que compara a diferença entre o valor esperado e o observado pelo modelo, indicando em que medida a árvore de decisão prediz os resultados corretamente.

Realizou-se análise descritiva das perdas dentárias segundo as variáveis independentes investigadas, assim como a associação destas por meio do teste qui-quadrado. As variáveis que apresentaram valores de $p \leq 0,20$ na análise bivariada foram inseridas no modelo de árvore de decisão por meio do Algoritmo Chi-squared Automatic Interaction Detector (CHAID).

\section{Resultados}

A Tabela 1 descreve as perdas dentárias em adultos segundo o capital social, características demográficas e socioeconômicas. A prevalência de cinco ou mais dentes perdidos foi de $42,5 \%$ ( $\mathrm{n}=$ 431). Em relação ao capital social, a maioria dos adultos $(n=628)$ pertenceu ao grupo de baixo capital social contra 339 adultos que representaram o grupo de alto capital social. A prevalência da perda dentária foi maior em pessoas com baixo capital social $(47,0 \%)$ quando comparadas aos do grupo de alto capital social $(36,3 \%), \mathrm{p}=$ 0,001 . Não houve diferença estatisticamente significante entre o número de dentes perdidos em homens $(\mathrm{n}=152 ; 40,5 \%)$ e mulheres $(\mathrm{n}=279$; $43,7 \%), p=0.320$. A perda dentária foi maior nos adultos mais velhos com idade entre 40 e 44

Tabela 1. Distribuição das perdas dentárias ( $\leq 04 ; \geq 5$ dentes perdidos) em adultos de $35-44$ anos, segundo o capital social, características demográficas e socioeconômicas. Municípios do entorno de Belo Horizonte, Minas Gerais, Brasil. 2010.

\begin{tabular}{|c|c|c|c|c|c|c|}
\hline \multirow[b]{2}{*}{ Variáveis } & \multicolumn{6}{|c|}{ Perdas dentárias } \\
\hline & Total & $\leq 04$ dentes & $\%$ & $\geq 05$ dentes & $\%$ & P-valor \\
\hline Prevalência & 1013 & 582 & 57,5 & 431 & 42,5 & - \\
\hline Capital Social [967] & & & & & & 0,001 \\
\hline Baixo & 628 & 333 & 53,0 & 295 & 47,0 & \\
\hline Alto & 339 & 216 & 63,7 & 123 & 36,3 & \\
\hline Gênero [1013] & & & & & & 0,320 \\
\hline Masculino & 375 & 223 & 59,5 & 152 & 40,5 & \\
\hline Feminino & 638 & 359 & 56,3 & 279 & 43,7 & \\
\hline Idade [1013] & & & & & & $<0,001$ \\
\hline $35-40$ & 500 & 351 & 70,2 & 149 & 29,8 & \\
\hline $41-44$ & 513 & 231 & 45,0 & 282 & 55,0 & \\
\hline Cor da pele [938] & & & & & & 0,221 \\
\hline Branca & 282 & 177 & 62,8 & 105 & 37,2 & \\
\hline Parda & 494 & 277 & 56,1 & 217 & 43,9 & \\
\hline Amarela & 30 & 20 & 66,7 & 10 & 33,3 & \\
\hline Preta & 126 & 73 & 57,9 & 53 & 42,1 & \\
\hline Indígena & 06 & 02 & 33,3 & 04 & 66,7 & \\
\hline Estado civil [1008] & & & & & & 0,134 \\
\hline Casado & 690 & 386 & 55,9 & 304 & 44,1 & \\
\hline Divorciado & 90 & 59 & 65,6 & 31 & 34,4 & \\
\hline Viúvo & 19 & 08 & 42,1 & 11 & 57,9 & \\
\hline Solteiro & 209 & 126 & 60,3 & 83 & 39,7 & \\
\hline Renda [966] & & & & & & 0,003 \\
\hline$>\mathrm{R} \$ 300$ & 454 & 282 & 62,1 & 172 & 37,9 & \\
\hline$\leq \mathrm{R} \$ 300$ & 512 & 270 & 52,7 & 242 & 47,3 & \\
\hline Escolaridade [1011] & & & & & & $<0,001$ \\
\hline Graduação & 82 & 61 & 74,4 & 21 & 25,6 & \\
\hline Ensino Médio & 340 & 226 & 66,5 & 114 & 33,5 & \\
\hline Ensino Fundamental & 568 & 284 & 50,0 & 284 & 50,0 & \\
\hline Nunca estudou & 21 & 10 & 47,6 & 11 & 52,4 & \\
\hline
\end{tabular}


anos ( $\mathrm{n}=282 ; 55,0 \%)$ do que nos mais jovens ( $\mathrm{n}$ $=149 ; 29,8 \%), \mathrm{p}<0,001$.

No que se refere às condições socioeconômicas, a cor da pele autorreferida $(\mathrm{p}=0,221)$ e o estado civil $(\mathrm{p}=1,134)$ não se associaram ao desfecho. Adultos indígenas (66,7\%), pardos (43,9\%) e pretos $(42,1 \%)$ perderam mais dentes do que os brancos $(37,2 \%)$ e amarelos $(33,3 \%)$. A perda dentária foi maior nos viúvos $(57,9 \%)$, casados $(44,1 \%)$ quando comparados aos solteiros $(39,7 \%)$ e divorciados $(34,4 \%)$ (Tabela 1$)$.

A renda per capita $(\mathrm{p}=0,003)$ e a escolaridade dos adultos se associaram às perdas dentárias sendo que os adultos de baixa renda $(\mathrm{n}=242$; $47,3 \%)$, analfabetos $(\mathrm{n}=11 ; 52,4 \%)$ ou que tinham cursado apenas o ensino fundamental ( $\mathrm{n}$ $=284 ; 50,0 \%$ ) perderam mais que quatro dentes quando comparados aos de renda mais alta $(\mathrm{n}=$ $172 ; 37,9 \%)$ e com maior nível de estudo formal ( $\mathrm{n}=21 ; 25,6 \%)$ (Tabela 1$)$.

A perda dentária de adultos pôde ser explicada pelo capital social $(\mathrm{p}<0,001)$, pela idade $(\mathrm{p}<$ $0,001)$, escolaridade $(p=0,022)$ e renda familiar per capita $(p=0,037)$. Formaram-se dois grupos distintos para explicar a perda dentária: baixo $(\mathrm{n}=628 ; 62,0 \%)$ e alto capital social $(\mathrm{n}=385$; $38,0 \%$ ). De maneira geral, a perda dentária ( $\geq 5$ dentes) foi mais prevalente em adultos com baixo capital social $(47,0 \%)$, com 40 a 44 anos de idade $(60,2 \%)$ e que cursaram apenas o ensino fundamental $(66,3 \%)$ quando comparados aos adultos de 35-39 anos (33,0\%) com renda familiar per capita maior que $\mathrm{R} \$ 300,00$. No grupo com alto capital social, adultos de 40-44 anos de idade perderam mais dentes $(46,1 \%)$ do que o grupo de 35-39 (24,7\%) (Figura 1).

\section{Discussão}

Foram analisados dados referentes a 1.013 adultos (taxa de resposta de 90,8\%). A mediana do número de dentes perdidos neste estudo foi igual a 04 (média $=5,3$ ), aproximando-se dos resultados do SB-Brasil 2010 cuja média de dentes perdidos foi de 7,3 em adultos de 35-44 anos de ida$\mathrm{de}^{3}$. É importante ressaltar a melhora da saúde bucal desse grupo etário uma vez que, no ano de 2003, a mediana de dentes perdidos em adultos brasileiros era igual a 11 dentes. O efeito de coorte da expansão/manutenção de fluoretação de águas e consumo de dentifrícios com flúor pode ter beneficiado a saúde bucal desses adultos ${ }^{10}$. Ademais, a política Nacional de Saúde Bucal foi estabelecida em 2004, ano posterior aos traba- lhos do SB-Brasil 2002-3. O país não contava com nenhuma política de Estado focada na saúde bucal, mas, sim, com ações pontuais e isoladas de promoção da saúde e prevenção das doenças e agravos. A implementação do Brasil Sorridente, o aumento das Equipes de Saúde Bucal (ESB) na Estratégia da Saúde da Família no Sistema Único de Saúde (SUS), assim como a ampliação da população coberta por essas equipes também podem ter contribuído para reduzir o número de dentes perdidos nos adultos. Em janeiro de 2011, 5.289 municípios brasileiros contavam com a Estratégia da Saúde da Família e 4.832 possuíam $\mathrm{ESB}^{23}$. Outro aspecto relevante no quesito assistencial é o funcionamento da média complexidade em Odontologia nos Centros de Especialidades Odontológicas (CEO) que oferecem, por exemplo, terapêutica endodôntica e protética para a população.

Em sentido ainda mais amplo, o crescimento econômico brasileiro, verificado a partir de 2003, foi o principal responsável pela redução da pobreza extrema no país. No ano de 1990, 25,6\% da população brasileira possuía renda domiciliar per capita abaixo da linha da pobreza; em 2008, $4,85 \%$ da população eram extremamente pobres ( $\leq \mathrm{R} \$ 70$ per capita) segundo critério internacional (1:20 brasileiros $)^{24}$. A redução da pobreza de um país está diretamente relacionada à melhoria das condições de vida das pessoas que, por sua vez, causa impacto positivo nos níveis de saúde da população.

O uso da análise de árvore de decisão é, relativamente, novo na saúde pública. Consiste em uma técnica onde há sistematização de dados para tomadas de decisões em condiçôes de incerteza, o que parece apropriado para ações de vigilância em saúde, pois pode ser direcionada tanto para condições clínicas individuais como populacionais. Questões como infecção pelo vírus da hepatite $\mathrm{C}$ em caminhoneiros brasileiros ${ }^{25}$, desfechos subjetivos como a qualidade de $v^{2} \mathrm{ad}^{26} \mathrm{e}$ a identificação de fatores de risco para reincidência do hábito de fumar $^{27}$ foram analisadas por meio de análises de árvores de decisão. A análise de árvore de decisão é considerada promissora para identificar populações de risco nas pesquisas em saúde pública ${ }^{28}$. No presente estudo, o perfil das perdas dentárias na população estudada pode ser explicado por diferenças entre o capital social, a idade, a escolaridade e a renda familiar per capita.

O capital social e a saúde é um tópico emergente na Saúde Pública ${ }^{29-31}$. Apesar de um dos primeiros estudos sobre o capital social e saúde ter sido publicado no final da década de $1990^{32}$, 


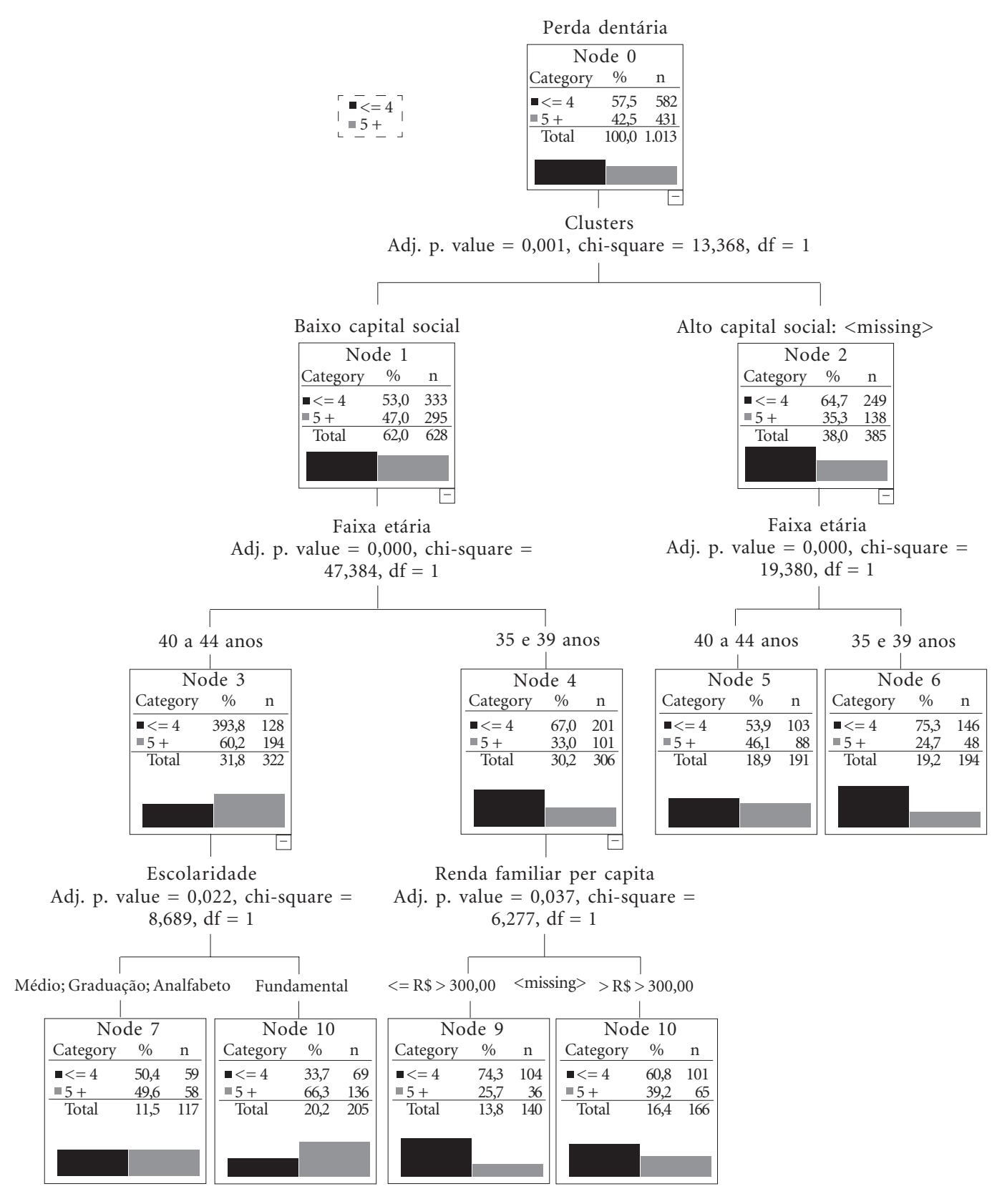

Figura 1. Análise multivariada por meio da árvore de decisão (CHAID) para a perda dentária em adultos de 35-44 anos, ajustada pelo capital social, fatores socioeconômicos e demográficos. Municípios do entorno de Belo Horizonte, Minas Gerais, Brasil. 2010.

as primeiras pesquisas odontológicas sobre esse tema datam de 2006 cujos desfechos investigados foram o traumatismo dentário em escolares $^{33}$ e a cárie dentária em adolescentes ${ }^{34,35}$. Até a presente data, poucos estudos investigaram o capital social e as perdas dentárias. Esses trabalhos foram conduzidos no Japão em população idosa ( $\geq 65$ anos) para analisar algumas dimensões do capital social: vertical (relações sociais hierárquicas) versus horizontal (relações sociais igualitárias) ${ }^{36}$, estrutural (o que as pessoas fazem) versus cognitivo (o que as pessoas pensam $)^{37}$ e o número de dentes remanescentes $(\geq 20 ; \leq 19)$. De maneira geral, os resultados desses trabalhos 
apontaram que o capital social teve efeitos benéficos para a manutenção de dentes naturais em idosos, porém com limitação de causalidade inerente ao desenho transversal desses estudos.

No presente estudo, os resultados apontaram que os adultos pertencentes ao grupo de "baixo capital social" (pessoas inseguras ou mais ou menos seguras em casa e que não participavam de nenhum grupo social - formal ou informal) perderam mais dentes do que os adultos do grupo com "alto capital social". Talvez seja um indício de vulnerabilidade social uma vez que essas pessoas residem em áreas com altos índices de criminalidade o que desencorajaria a interação social. Mais do que isso, o local onde o indivíduo vive influencia a condição de saúde dos moradores, independente das características individuais de cada uma dessas pessoas ${ }^{38}$. Moradores de bairros mais pobres autoavaliam pior a saúde bucal e perdem mais dentes quando comparados aos que vivem em bairros mais ricos, independentemente do sexo, idade, escolaridade e renda familiar ${ }^{39}$. A variação do número de dentes perdidos entre moradores de bairros mais abastados é inexpressiva, apesar das características socioeconômicas individuais. Porém, em bairros pobres, a variação das perdas dentárias entre os moradores é explicada pela renda per capita ${ }^{40}$.

Segundo a análise de árvore de decisão do presente estudo, observou-se que possuir "alto capital social" pode ter atenuado a influência da renda e da escolaridade na distribuição das perdas dentárias nos adultos independentemente da idade. A potencial influência do capital social em atenuar o efeito da condição socioeconômica sobre a saúde também foi verificado por Elgar et $\mathrm{al}^{41}$. Estes autores investigaram se a exposição de adolescentes ao capital social da vizinhança (indicadores: confiança, coesão e cooperação) reduziria diferenças socioeconômicas sobre saúde física e mental desses jovens. Concluíram que o efeito da condição socioeconômica dos adolescentes na saúde dos mesmos variou de acordo com o nível de exposição do capital social do bairro ou vizinhança ${ }^{41}$

Apesar da diferença substancial entre o capital social (produto das relações sociais) e a condição socioeconômica (posição que uma pessoa ocupa na sociedade de acordo com o nível de escolaridade e renda), é importante ressaltar que, assim como na condição socioeconômica ${ }^{42}$, o que importa para a saúde não é apenas o total de capital social disponível em uma sociedade, mas, sim a maneira como os diferentes níveis estão distribuídos entre as pessoas.
Uma das hipóteses sobre a relação entre o capital social e a saúde é a influência de comportamentos saudáveis por meio de difusão rápida de informação, aumentando a probabilidade das pessoas acatarem recomendações positivas para uma vida mais saudável ${ }^{43}$. Neste estudo, a participação em grupos sociais, formais ou informais, pôde ter possibilitado troca de informações sobre saúde bucal e não adoção de comportamentos de risco para as perdas dentárias como o tabagismo, por exemplo. Além disso, é posto que o capital social facilite o acesso aos serviços de saúde ${ }^{44}$. Nesse sentido, pessoas do grupo de alto capital social podem ter tido mais chances de conseguir tratar a doença cárie instalada, evitando a perda do elemento dental, por meio do suporte social existente nos grupos nos quais disseram fazer parte.

A distribuição das perdas dentárias variou de acordo com a idade em ambos os grupos de capital social. Adultos de 40-44 anos perderam mais dentes do que os de 35-39 anos. Tal direção de associação foi esperada visto que quanto mais velho for o indivíduo, maior será o tempo que o dente ficará na cavidade bucal exposto aos fatores de riscos de doenças e ou agravos ${ }^{45}$. Há de se ressaltar que, apesar da mesma direção de associação, a prevalência da perda dentária entre faixas etárias iguais variou segundo o capital social. Por exemplo, adultos de 40-44 anos do grupo de baixo capital social perderam mais dentes $(60,2 \%)$ do que adultos de 40-44 anos do grupo de alto capital social (35,3\%). A renda e a escolaridade não interferiram na distribuição das perdas dentárias dentro do grupo de alto capital, independente do grupo etário. Em contrapartida, essas mesmas variáveis tiveram diferentes padrões de associação com o desfecho de acordo com a idade dos adultos do grupo de baixo capital social. A escolaridade se associou à perda dentária na faixa etária de 40-44 anos enquanto a renda familiar per capita foi associada à perda dentária em adultos de 35-39 anos de idade.

O presente estudo apresenta limitações. Como se trata de um estudo transversal, nenhuma relação de causalidade poderá ser inferida a partir desses resultados. Além disso, foram investigadas apenas características individuais. Seria desejável a inclusão de variáveis contextuais para que o perfil da perda dentária na população estudada fosse traçado de uma maneira mais ampla, como desejável nos estudos de determinantes sociais.

Apesar da tentativa de criar a variável binária de capital social (alto; baixo) por meio de análise 
de segmentação, apenas dois dos quatro indicadores de capital social resultaram em combinação satisfatória para tal (participação em grupos e segurança com relação ao crime e à violência quando sozinho em casa). Assim, a mensuração do capital social pode ter falhado por não contemplar outros aspectos e dimensões desse determinante social. Seria desejável a inclusão de variáveis contextuais referentes ao capital social no nível coletivo.

Outra limitação se refere à própria análise de árvore de decisão uma vez que, apesar de traçar o perfil do desfecho, não mede a magnitude das associações e nem o quanto a variação entre as categorias de algumas variáveis pode ter contribuído com a perda da associação de outras. Porém, cumpriu o objetivo proposto cujo foco foi obter uma descrição das perdas dentárias em adultos, mapeando o agravo e os determinantes selecionados. Além disso, os resultados podem nortear ações de promoção da saúde ou de prevenção das perdas dentárias na população estudada. Outra questão é a possibilidade da replicação da análise de árvore de decisão em outros estudos científicos na área da saúde assim como em trabalhos desenvolvidos no próprio Sistema Único de Saúde como um suporte para a tomada de decisões de gestores e demais envolvidos no setor saúde.

O presente estudo contribuiu com o avanço no conhecimento sobre o padrão da distribuição das perdas dentárias em adultos por dois motivos. Primeiro, trata-se de uma população pouco estudada devido às dificuldades de se chegar à população adulta (ex.: ausência da residência em horário comercial, local de trabalho como barreira à condução de pesquisas). Segundo, este é um dos primeiros estudos a unir algum aspecto do capital social aos fatores já bem estabelecidos na literatura, como socioeconômicos e demográficos, para traçar um perfil de um agravo que acomete tantos brasileiros como a perda dentária.

\section{Conclusão}

Conclui-se que, no nível individual, o capital social, a idade e a renda explicaram o perfil das perdas dentárias. A presença de capital social pode ter atenuado o efeito negativo dos fatores socioeconômicos na população estudada.
Frente ao grande desafio da equidade em saúde bucal no Brasil, os resultados deste estudo poderão contribuir com o Sistema Único de Saúde, uma vez que as relações sociais podem ser alternativas auxiliares de ações de promoção da saúde bucal dos brasileiros, seja por meio da canalização do capital social para impulsionar ações coletivas, no mapeamento mais amplo de grupos vulneráveis, ou, ainda, impulsionado novos estudos sobre o capital social como um importante determinante do processo saúde-doença. Investigar a possível associação entre capital social e a saúde das pessoas é uma importante contribuição para o desafio do combate às iniquidades em saúde no país.

\section{Colaboradores}

CM Borges e ACV Campos idealizaram o estudo; ACV Campos realizou a análise estatística; CM Borges conduziu o estudo e a redação do manuscrito. AMD Vargas e EF Ferreira contribuíram com a revisão final e crítica do manuscrito.

\section{Agradecimentos}

À Fundação de Amparo à Pesquisa do Estado de Minas Gerais (FAPEMIG) pelo financiamento e à Coordenação de Aperfeiçoamento de Pessoal de Nível superior (CAPES) pela bolsa de doutorado da primeira autora. Às pesquisadoras Aline Mendes Silva, Maria de Lourdes Carvalho e Simone Costa Melo pelas valiosas discussões ao longo de todo o estudo. 


\section{Referências}

1. Brasil. Ministério da Saúde (MS). Divisão Nacional de Saúde Bucal. Levantamento Epidemiológico em Saúde Bucal: Brasil, zona urbana. Brasília: MS; 1988.

2. Brasil. Ministério da Saúde (MS). Secretaria de Atenção à Saúde. Departamento de Atenção Básica. Projeto SB Brasil 2003: condições de saúde bucal da população brasileira 2002-2003: resultados principais. Brasília: MS; 2004.

3. Brasil. Ministério da Saúde (MS). Secretaria de Atenção a Saúde. Departamento de Atenção Básica. Coordenação de Saúde Bucal. Projeto SB Brasil 2010. Nota para a imprensa. Brasília: MS; 2011.

4. Jovino-Silveira RC, Caldas Júnior AF, Cabral HA, Gusmão ES. Primary reason for tooth extraction in a Brazilian adult population. Oral Health Prev Dent 2005; 3(3):151-157.

5. Cimões R, Caldas-Júnior AF, Souza EHA, Gusmão ES. Influência de classe social nas razões clínicas das perdas dentárias. Cien Saude Colet 2007; 12(6): 1691-1696.

6. Petersen PE. The World Oral Health Report 2003. Continuous improvement of oral health in the 21st century: the approach of the WHO Global Oral Health Programme. Community Dent Oral Epidemiol 2003; 31(Supl. 1):03-24

7. Petersen PE. Tobacco and oral health: the role of the World Health Organization. Oral Health Prev Dent 2003b; 1(4):309-315.

8. Caldas-Júnior AF. Reasons for tooth extraction in a Brazilian population. Int Dent J 2000; 50(5):267-273.

9. Cabral ED, Caldas Júnior AF, Cabral HA. Influence of the patients race on the dentist's decision to extract or retain a decayed tooth. Community Dent Oral Epidemiol 2005; 33(6):461-466.

10. Barbato PR, Nagano HCM, Zanchet FN, Boing AF, Peres MA. Perdas dentárias e fatores sociais, demográficos e de serviços associados em adultos brasileiros: uma análise dos dados do Estudo Epidemiológico Nacional (Projeto SB Brasil 2002-2003). Cad Saude Publica 2007; 23(8):1803-1814

11. Moreira RS, Nico LS, Barrozo LV, Pereira JC. Tooth loss in Brazilian middle-aged adults: multilevel effects. Acta Odontol Scand 2010; 68(5):269-277.

12. Silva DD, Rihs LB, Sousa MLR. Fatores associados à presença de dentes em adultos de São Paulo, Brasil. Cad Saude Publica 2009; 25(11):2407-2418.

13. De Souza e Silva ME, de Magalhães CS, Ferreira EF. Complete removable prostheses: from expectation to (dis) satisfaction. Gerodontology 2009; 26(2):143 149 .

14. Gerritsen AE, Allen PF, Witter DJ, Bronkhorst EM, Creugers NH. Tooth loss and oral health-related quality of life: a systematic review and meta-analysis. Health Qual Life Outcomes 2010; 8:126.

15. Ferreira AAA, Piuvezam G, Werner CWA, Alves MSCF. The toothache and tooth loss: social representation of oral care. Cienc Saude Colet 2006; 11(1):211-218.

16. Coleman J. Social capital in the creation of human capital. Am J Sociology 1988; 94(Supl.):95-120.

17. Putnam R. Making democracy work: civic transitions in modern Italy. New Jersey: Princeton University Press; 1993.
18. Aida J, Hanibuchi T, Nakade M, Hirai H, Osaka K, Kondo K. The different effects of vertical social capital and horizontal social capital on dental status: multilevel analysis. Soc Sci Med 2009; 69(4):512-518.

19. Frazão P, Antunes JLF, Narvai PC. Perda dentária precoce em adultos de 35-44 anos de idade. Estado de São Paulo, Brasil, 1998. Rev Bras Epidemiol 2003; 6(1):49-57

20. World Health Organization (WHO). Oral Health Surveys: basic methods. $4^{\text {a }}$ ed. Geneva: WHO Press; 1997.

21. Grootaert C, Narayan D, Jones VN, Woolcock M. Measuring social capital: an integrated questionnaire. Washington: World Bank; 2004

22. Hair JF, Black WC, Babin JB, Anderson RE, Tatham RL. Segmentation Analysis. In: Hair JF, Black WC Babin JB, Anderson RE, Tatham RL, organizadores. Multivariate Data Analysis. New Jersey: PrenticeHall; 2006. p. 427-481.

23. Brasil. Ministério da Saúde (MS). Sistema de Informação da Atenção Básica. [acessado 2014 abr 19]. Disponível em: http://siab.datasus.gov.br/

24. Instituto Econômico de Pesquisa Aplicada (Ipea) Objetivos de desenvolvimento do milênio: relatório nacional de acompanhamento. Brasília: Ipea; 2010.

25. Freitas NR, Teles AS, Matos AM, Lopes CLR, Reis NRS, Espírito-Santo MP, Lampe E, Martins RMB. Hepatitis $C$ virus infection in Brazilian long-distance truck drivers. Virol J 2010; 7:205.

26. Campolina AG, Ciconelli RM. Qualidade de vida e medidas de utilidade: parâmetros clínicos para as tomadas de decisão em saúde. Rev Pan Salud Publica 2006; 19(2):128-136

27. Piper ME, Loh W, Smith S, Japuntich S, Barker T. Using Decision Tree Analysis to Identify Risk Factors for Relapse to Smoking. Inform Healthcare 2011; 46(4):492-510.

28. Lemon SC, Roy J, Clark MA, Friedmann PD, Rakowski W. Classification and regression tree analysis in public health: methodological review and comparison with logistic regression. Ann Behav Med 2003; 26(3):172-181.

29. De Silva MJ, McKenzie K, Harpham T, Huttly SR. Social capital and mental illness: a systematic review. J Epidemiol Community Health 2005; 59(8):619627.

30. Kripper CE, Sapag JC. Social capital and health in Latin America and the Caribbean: a systematic review. Rev Pan Salud Publica 2009; 25(2):162-170.

31. Hibino Y, Takaki J, Ogino K, Kambayashi Y, Hitomi Y, Shibata A, Nakamura H. The relationship between social capital and self-rated health in a Japanese population: a multilevel analysis. Environ Health Prev Med 2012; 17(1):44-52.

32. Kawachi I, Kennedy BP, Lochner K, Prothrow-Stith D. Social capital, income inequality, and mortality. Am J Public Health 1997; 87(9):1491-1498.

33. Moysés SJ, Moysés ST, McCarthy M, Sheiham A. Intra-urban differentials in child dental trauma in relation to healthy cities policies in Curitiba, Brazil. Health Place 2006; 12(1):48-64. 
34. Pattussi MP, Hardy R, Sheiham A. Neighborhood social capital and dental injuries in Brazilian adolescents. Am J Public Health 2006; 96(8):1462-1468.

35. Pattussi MP, Hardy R, Sheiham A. The potential impact of neighborhood empowerment on dental caries among adolescents. Community Dent Oral Epidemiol 2006; 34(5):344-350.

36. Aida J, Hanicuchi T, Nakade Miyo, Hirai H, Osaka $\mathrm{K}$, Kondo K. The different effects of vertical and horizontal social capital on dental status: a multilevel analysis. Soc Sci Med 2009; 69(4):512-518.

37. Aida J, Kuriyama S, Ohmori-Matsuda K, Hozawa A, Oska K, Tsuji I. The association between neighborhood social capital and self-reported dentate status in elderly Japanese - The Ohsaki Cohort 2006 study. Community Dent Oral Epidemiol 2011; 39(3): 239-249.

38. Riva M, Gauvin L, Barnett TA. Toward the next generation of research into small area effects on health: a synthesis of multilevel investigations published since July 1998. J Epidemiol Community Health 2007; 61(10):853-861.

39. Turrell G, Sanders AE, Slade GD, Spencer AJ, Marcenes $\mathrm{W}$. The independent contribution of neighborhood disadvantage and individual-level socioeconomic position to self-reported oral health: a multilevel analysis. Community Dent Oral Epidemiol 2007; 35(3):195-206.

40. Sanders AE, Turrell G, Slade GD. Affluent neighborhoods reduce excess risk for tooth loss among the poor. J Dent Res 2008; 87(10):969-973.

41. Elgar FJ, Trites SJ, Boyce W. Social capital reduces socio-economic differences in child health: evidence from the Canadian Health Behaviour in School-Aged Children study. Can J Public Health 2010; 101(Supl. 3):S23-27.

42. Daniels N, Kennedy B, Kawachi I. Justice is good for our health. In: Daniels N, Kennedy B, Kawachi I Kawachi I. Is inequality bad for our health? Boston: Beacon Press; 2000. p. 3-33.

43. Berkman L. Social cohesion, social capital, and health. In: Berkman L, Kawachi I, organizadores. Social Epidemiology. New York: Oxford University Press; 2000. p. 174-190.

44. Derose KP, Varda DM. Social capital and health care access: a systematic review. Med Care Res Rev 2009; 66(3):272-306

45. Thomson WM, Poulton R, Milne BJ, Caspi A Broughton JR, Ayers KM. Socioeconomic inequalities in oral health in childhood and adulthood in a birth cohort. Community Dent Oral Epidemiol 2004; 32(5):345-353.

Artigo apresentado em 15/03/2013

Aprovado em 25/03/2013

Versão final apresentada em 26/03/2013 